Supporting information for

\title{
Basic structure-independent equations of kinetic performance of columns in liquid chromatography
}

Leonid M. Blumberg*

Advachrom, P.O. Box 1243, Wilmington, DE 19801, USA

leon@advachrom.com.

Table of Contents

van Deemter and Knox plate height equations 2

Beyond generalized Carr-Matule problem 4

$\begin{array}{ll}\text { References } & 6\end{array}$ 


\section{van Deemter and Knox plate height equations}

Several dependencies of plate height $(H)$ on solvent velocity $(u)$ in LC columns packed with approximately identical spherical particles are known ${ }^{1-5}$. The most widely used are the van Deemter ${ }^{1}$ and the Knox ${ }^{4-5}$ equations. In dimensionless form, they can be expressed as:

$h=A+\frac{B}{v}+C v, \quad($ van Deemter et al. $)$

$h=A v^{1 / 3}+\frac{B}{v}+C v, \quad(\operatorname{Knox})$

where $h$ and $v$ are, respectively, the dimensionless plate height and dimensionless velocity defined as $h=H / d_{\mathrm{p}}$ and $v=u d_{\mathrm{p}} / D_{\mathrm{m}}$. In these equations, $d_{\mathrm{p}}$ and $D_{\mathrm{m}}$ are, respectively, the particle diameter and the solute molecular diffusivity in the solvent. There is optimal solvent velocity at which the plate height has minimum. Their dimensionless values are $v_{\mathrm{opt}}$ and $h_{\mathrm{min}}$. The van Deemter equation has analytical (closed form) solutions for $v_{\mathrm{opt}}$ and $h_{\mathrm{min}}$ :

$v_{\mathrm{opt}}=\sqrt{B / C}, \quad h_{\min }=A+2 \sqrt{B C}, \quad($ van Deemter et al. $)$

Also important is the ratio

$a=A / h_{\min }=A d_{\mathrm{p}} / H_{\min }$

-the eddy diffusion component of the plate height as fraction of the minimal plate height.

Unfortunately, the analytical solutions for $v_{\mathrm{opt}}$ and $h_{\min }$ of Knox equation do not exist. This raises question of using van Deemter equation instead of the Knox equation when possible. Evaluating the errors of this replacement, on can recognize that both van Deemter and Knox equations are empirical ones and that their $(A, B, C)$ parameters are typically found from the curve-fitting to experimental data calculated from the peak retention times and standard deviations $(\sigma)$ at different solvent velocities usually below and above their optimum. The accuracy of evaluation of $\sigma$ from the peak width measurements is relatively low (typically worse than 5-10\%).

Knox mentioned ${ }^{5}$ the ABC-parameters of his plate height equation for a "reasonably well packed column", and a one with "exceptional" packing. The least square fitting of van Deemter equation into these Knox equations for $0.5<v<50$ are considered below (Figure S1).

Knox's dimensionless plate height equation for "reasonably well packed column" (Figure S1a): 
$h=v^{1 / 3}+\frac{2}{v}+0.1 v$

Its van Deemter least square approximation and its optimal parameters are:

$h=1.605+\frac{1.406}{v}+0.149 v$

$v_{\mathrm{opt}}=3.07, \quad h_{\min }=2.52, \quad a=0.637$

Knox's dimensionless plate height equation for "exceptional" packing (Figure S1b):

$h=0.5 v^{1 / 3}+\frac{2}{v}+0.05 v$

Its van Deemter least square approximation and its optimal parameters:

$h=0.803+\frac{1.703}{v}+0.0746 v$

$v_{\mathrm{opt}}=4.78, \quad h_{\min }=1.52, \quad a=0.53$ 

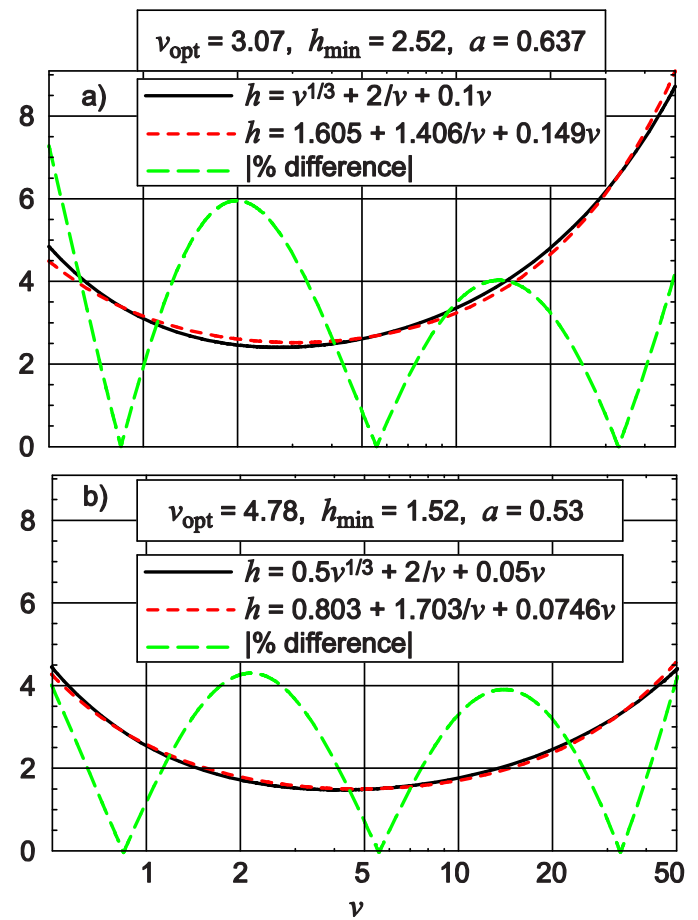

Figure S1. Least square approximations, eqs (S6) and (S9), of Knox equations, eqs (S5) and (S8), by Deemter equation, eq (S1).

\section{Beyond generalized Carr-Matule problem}

The following two equations were derived in the main text. One expresses the ratio $t_{\mathrm{M}} / t_{\mathrm{M} \text {,ref }}$ as a

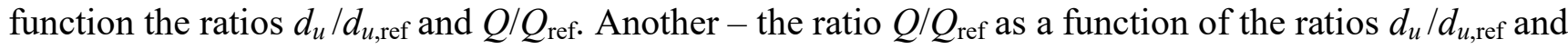
$t_{\mathrm{M}} / t_{\mathrm{M}, \mathrm{ref}}$ :

$$
\begin{aligned}
& \frac{t_{\mathrm{M}}}{t_{\mathrm{M}, \mathrm{opt}}}=\left((1-a)\left(\frac{d_{u}}{d_{u, \mathrm{ref}}}\right)^{2} \frac{Q}{Q_{\mathrm{ref}}} /\left(\sqrt{\left(\frac{Q}{Q_{\mathrm{ref}}}\right)^{2}+2(1-a)\left(\left(\frac{d_{u}}{d_{u, \mathrm{ref}}}\right)^{2}-\left(\frac{Q}{Q_{\mathrm{ref}}}\right)^{2}\right)}-\frac{a Q}{Q_{\mathrm{ref}}}\right)\right)^{2} \\
& \frac{Q}{Q_{\mathrm{ref}}}=\frac{d_{u}}{d_{u, \mathrm{ref}}} \sqrt{\frac{2 t_{\mathrm{M}}}{t_{\mathrm{M}, \mathrm{opt}}} /\left(\frac{d_{u}^{4}}{d_{u, \text { ref }}^{4}}+\frac{t_{\mathrm{M}}}{t_{\mathrm{M}, \mathrm{opt}}}-a\left(\sqrt{\left.\left.\frac{t_{\mathrm{M}}}{t_{\mathrm{M}, \mathrm{opt}}}-\frac{d_{u}^{2}}{d_{u, \text { ref }}^{2}}\right)^{2}\right)}\right.\right.}
\end{aligned}
$$

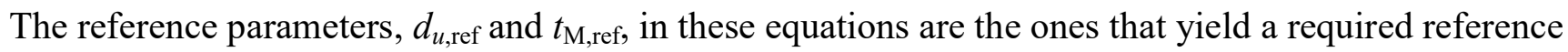
$Q\left(Q_{\mathrm{ref}}\right)$ in a column optimized at a predetermined fixed pressure $\Delta p$. Parameters $Q$ and $t_{\mathrm{M}}$ result from variation of $d_{u}$ and the column length $(L)$ while keeping $\Delta p$ fixed. 

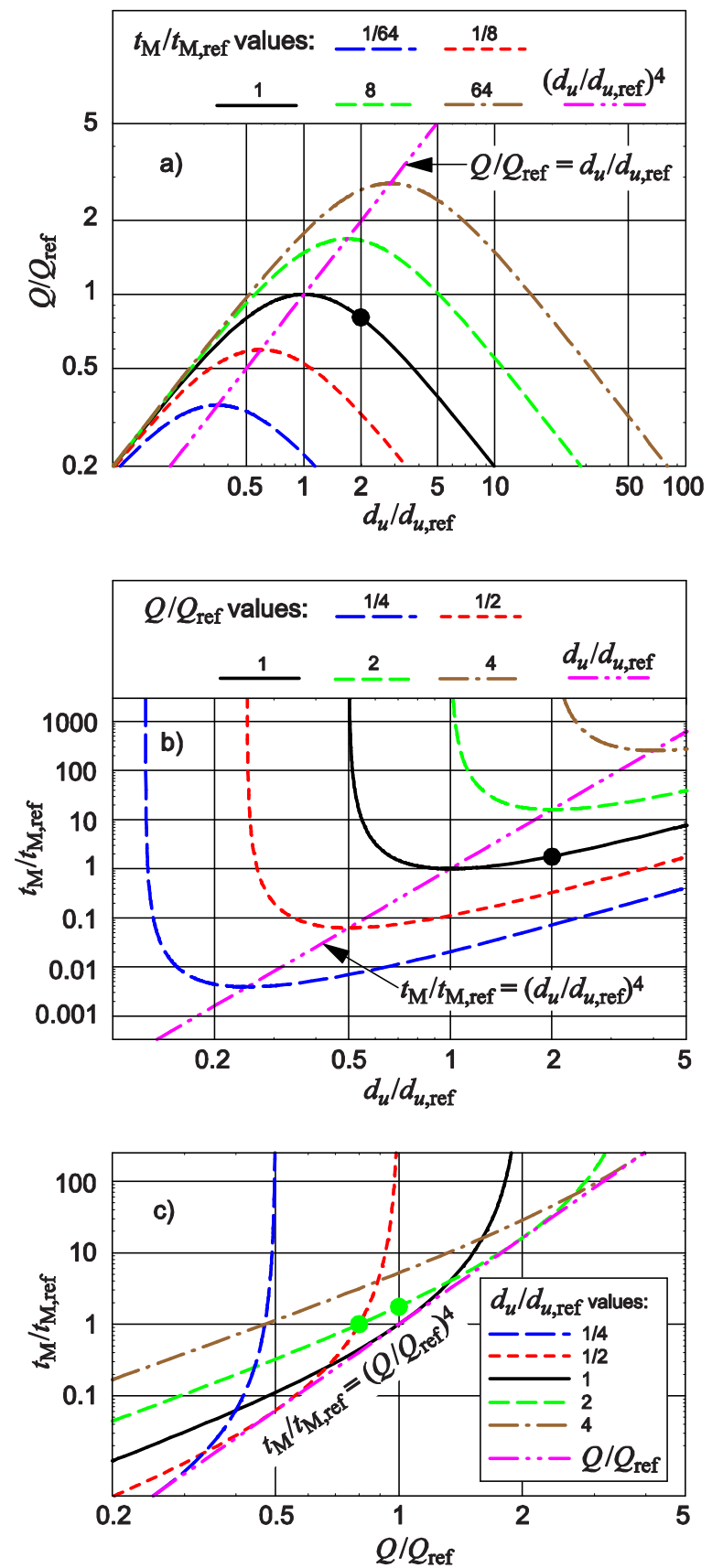

Figure S2. Effects of changes in velo-diameter $\left(d_{u}\right)$ on transport efficiency $(Q)$ and void time $\left(t_{\mathrm{M}}\right)$, eqs (S13) and (S14), at fixed pressure $(\Delta p)$ in a column with $a=0.5$. For each $d_{u}$, the column length $(L)$ is chosen to yield $t_{\mathrm{M}} / t_{\mathrm{M} \text {,ref }}$ values in the legends of (a), or $Q / Q_{\text {ref }}$ values in the legends of (b). No conditions are imposed on $(L)$ in (c). The dots mark the conditions of the Carr-Matula problem $\left(d_{u}=2 d_{u, \text { ref, }}\right.$ and either $t_{\mathrm{M}}=t_{\mathrm{M} \text {,ref }}$ or $\left.Q=Q_{\mathrm{ref}}\right)$.

Eq. (S11) can be used for finding the change in $t_{\mathrm{M}}$ relative to $t_{\mathrm{M} \text {,ref }}$ when a required departure of $d_{u}$ from $d_{u \text {,ref }}$ is accompanied by a required departure of $Q$ from $Q_{\text {ref. }}$ Alternatively, eq. (S12) can be used for finding the change in $Q$ relative to $Q_{\text {ref }}$ when a required departure of $d_{u}$ from $d_{u \text {,ref }}$ is accompanied 


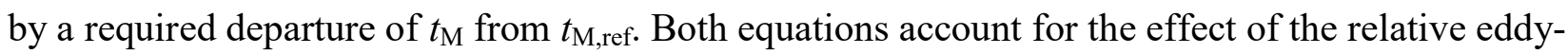
diffusion factor $a=A / H_{\min }$ on the changes.

Suppose that $a=0.5$ ( $A$-term in van Deemter equation is half of $H_{\min }$ ). Eqs. (S11) and (S12) become (Figure S2):

$$
\begin{aligned}
& \frac{t_{\mathrm{M}}}{t_{\mathrm{M}, \mathrm{opt}}}=\left(\frac{\left(d_{u} / d_{u, \text { ref }}\right)^{2} Q / Q_{\mathrm{ref}}}{2 d_{u} / d_{u, \text { ref }}-Q / Q_{\mathrm{ref}}}\right)^{2}, a=0.5 \\
& \frac{Q}{Q_{\mathrm{ref}}}=\frac{2\left(d_{u} / d_{u, \text { ref }}\right) \sqrt{t_{\mathrm{M}} / t_{\mathrm{M}, \mathrm{opt}}}}{\left(d_{u} / d_{u, \mathrm{ref}}\right)^{2}+\sqrt{t_{\mathrm{M}} / t_{\mathrm{M}, \mathrm{opt}}}}, a=0.5
\end{aligned}
$$

\section{References}

(1) Van Deemter, J. J. J.; Zuiderweg, F. J.; Klinkenberg, A. Longitudinal Diffusion and Resistance to Mass Transfer as Causes of Nonideality in Chromatography, Chem. Eng. Sci. 1956, 5, 271-289.

(2) Giddings, J. C., Dynamics of Chromatography; Marcel Dekker, Inc.: New York; 1965.

(3) Giddings, J. C., Unified Separation Science; Wiley: New York; 1991.

(4) Knox, J. H. Practical Aspects of Lc Theory, J. Chromatogr. Sci. 1977, 15, 352-354.

(5) Knox, J. H. Theoretical Aspects of Lc with Packed and Open Small-Bore Columns, J. Chromatogr. Sci. 1980, 18, 453-481. 